\title{
Monte Carlo simulations of the critical properties of the restricted primitive model
}

\author{
J.-M.Caillol \\ Laboratoire de Physique Théorique \\ UMR 8267, Bât. 210 \\ Université de Paris-Sud \\ 91405 Orsay Cedex, France
}

Received August 16, 2004, in final form August 25, 2004

\begin{abstract}
Recent Monte Carlo simulations of the critical point of the restricted primitive model for ionic solutions are reported. Only the continuum version of the model is considered. A finite size scaling analysis based in the BruceWilding procedure gives critical exponents in agreement with those of the three-dimensional Ising universality class. An anomaly in the scaling of the specific heat with system size is pointed out.
\end{abstract}

Key words: primitive model, ionic criticality, Monte Carlo simulation

PACS: 05.70.Jk, 05.10.-a, 64.70.Fx, 61.20.-p

\section{Introduction}

The primitive model (PM) for electrolytes, molten salts, colloids, etc is a mixture of $M$ species of charged hard spheres living either on a lattice or within a continuous volume of real space. In this paper we shall focus only on the off-lattice version of the model. The simplest version of the PM consists of a binary mixture (i.e. $M=2$ ) of positive and negative charged hard spheres $\pm q$ all with the same diameter $\sigma$. Under this form the model which is thought to be the prototype of many ionic fluids has been christened the restricted primitive model (RPM). A thermodynamic state of the RPM is entirely specified by a reduced density $\rho^{*}=N \sigma^{3} / V$ ( $N$ number of ions, $V$ volume) and a reduced temperature $T^{*}=k T \sigma / q^{2}$ ( $k$ Boltzmann's constant).

The RPM undergoes a liquid-vapor transition which has been studied extensively these last past years by means of Monte Carlo (MC) simulations and various theoretical approaches. In particular the behavior of the system at its critical point (CP) has been the subject of a huge amount of numerical and theoretical studies. The question is obviously of great importance since it is reasonable to assume that real electrolytes - or at least a large class of them- and the RPM belong to the same 
universality class which dictates a similar critical behavior.

It is perhaps the right place to note that the main feature of ionic solutions is that the pair potential between two ions $i$ and $j$ at a distance $r_{i j}=\left|\mathbf{r}_{i}-\mathbf{r}_{j}\right|$ which reads as

$$
\begin{aligned}
& v\left(r_{i j}\right)=\frac{q_{i} q_{j}}{r_{i j}} \text { for } r_{i j}>\sigma, \\
& v\left(r_{i j}\right)=+\infty \text { for } r_{i j}<\sigma
\end{aligned}
$$

is a long range interaction. This fact would suggest classical (mean field) behavior, whereas the well-known screening of the interactions pleads in favor of an Ising-like criticality typical of systems with short range interactions.

On the experimental side it seems well established now that for many real electrolytes apparent mean field behavior applies with sharp crossover (much sharper than in nonionic fluids) to Ising criticality close to the critical temperature [1].

At the moment there exists no convincing theoretical proof showing that the RPM belongs to the Ising universality class [2-5] and only sophisticated Monte Carlo simulations can support this claim. Most numerical studies of the CP of the off-lattice version of the RPM were performed by the Orsay group and I would like to review our contributions towards a better understanding of the critical properties of this model in the lines below.

\section{A brief historical survey}

Quite generally, a single component fluid will undergo a liquid vapor transition if the pair potential which is assumed to represent the molecular interactions is (sufficiently) attractive at large distances. From this point of view the situation is not so clear in the case of the RPM (cf equation (1.1)) and the very existence of the transition is not guaranteed. Several studies were necessary to clarify this point and a brief historical survey is worthwhile.

The first evidence that the RPM actually undergoes a liquid-vapor transition can be tracked back to two papers of Chasovkikh and Vorontsov-Vel' Yaminov (CVVY) published as soon as in 1976 [6,7]. These authors performed isobaric MC simulations and found a transition with a CP located at $T_{\mathrm{c}}^{*}=0.095, \rho_{\mathrm{c}}^{*}=0.24$. Several years after (in 1991) Valleau studied three isotherms of the RPM with his method of the density scaling $\mathrm{MC}$ and obtained a different location for the $\mathrm{CP}$, namely $T_{\mathrm{c}}^{*}=0.07$, $\rho_{\mathrm{c}}^{*}=0.07$ [8]. Subsequently (in 1992) Panagiotopoulos [9] obtained still different results, i.e. $T_{\mathrm{c}}^{*}=0.056, \rho_{\mathrm{c}}^{*}=0.04$, by performing $\mathrm{MC}$ simulations in the Gibbs ensemble (GE), at the moment a powerful new method of simulation which he had invented a little bit earlier [10]. Subsequent GE simulations using an improved biased MC sampling [11] yielded Panagiotopoulos and Orkoulas to the new estimate $T_{\mathrm{c}}^{*}=0.053, \rho_{\mathrm{c}}^{*}=0.025$. Finally, making use myself of the Gibbs ensemble combined with the use of hyperspherical geometries I obtained rather $T_{\mathrm{c}}^{*}=0.057, \rho_{\mathrm{c}}^{*}=0.04$ $[12,13]$. 
Commenting on this striking dispersion of the MC data Prof M. Fisher talked once of the "sad street of numerical simulations". This was in 1999, at the SCCS conference, St Malo, France and, at this point of the story, I must agree with him retrospectively. However many advances have been done since. Before giving an account of these new achievements some comments are in order.

(i) All the MC studies confirm the existence of a liquid vapor transition for the RPM. It seems to take place at unusually low densities and temperatures. Caillol and Weis give further support for such a low critical temperature [14]. Moreover it turns out that the coexistence curve is very dissymmetric $[9,11,12]$.

(ii) The MC simulation of ionic systems is a numerical challenge due to the long range of Coulomb potential. In order to deal with this, some caution is needed. Thus, in the case of MC simulations performed in a cubic box with periodic boundary conditions (PBC), one must use Ewald potentials in order to obtain the correct physics [15-17]. The point is that the Ewald potential is the solution of Poisson equation in a cubico-periodical geometry [17] and many properties of ionic fluids (electro-neutrality, screening, etc) are a consequence of this fact. In their MC simulations CVVY and Valleau considered truncated Coulomb potentials and very small samples of $N=32$ particles which yields quantitatively wrong results. By contrast the data of Panagiotopoulos et al. $[9,11]$ are more reliable since Ewald sums have been used. The same remark apply to my simulations which were performed on a 4D sphere (a hypersphere for short) by considering interactions obtained by solving Poisson equation in this geometry. This alternative method of simulation is therefore also indisputably correct, moreover it is much more efficient. The rough agreement observed between the simulations of $[9,11]$ and [12] both involving the same number of ions, i.e. $N=512$, is therefore not fortuitous.

(iii) None of the above mentioned studies took correctly into account finite size effects which are of an overwhelming importance near a CP. These effects affect the behavior of finite systems as soon as the correlation length of the critical density fluctuations is of the same order of magnitude as the size of the simulation box. In the simulations $[9,11,12]$ some "apparent" critical temperature $T_{\mathrm{c}}^{*}$ has been measured which could be very different from its infinite volume limit $T_{\mathrm{c}}^{*}(\infty)$.

In order to extract from MC simulations the critical behavior of the RPM in the thermodynamic limit (i.e. the critical exponents) as well as the infinite volume limit of $T_{\mathrm{c}}^{*}$ and $\rho_{\mathrm{c}}^{*}$ it is necessary to perform an analysis of the MC data in the framework of the finite size scaling (fss) theory which is part of the renormalization group (RG) theory $[18,19]$. In this approach one needs to work in the Grand Canonical (GC) ensemble rather than in the Gibbs ensemble which is ill adapted for a fss analysis. Subsequent MC simulations on the RPM were thus all performed in this ensemble. Panagiotopoulos and coworkers turned their attention to the lattice version of the RPM whereas the Orsay group continued to work on its off-lattice version. 


\section{Finite size scaling analysis of MC data}

\subsection{Scaling fields and operators}

Starting with the seminal work of Bruce and Wilding (BW) [20-22] simulation results for the critical behavior of fluids have customarily been analyzed along the lines of the so-called revised scaling theory of Rehr and Mermin [23]. In this approach one first defines scaling fields and operators aimed at restoring the particle-hole symmetry and therefore to map the the fluid onto a magnetic sytem with Ising-like symmetry.

The two relevant scaling fields $h$ (the strong ordering field) and $\tau$ (the weak thermal field) are assumed to be linear combinations of deviations from their critical values of the chemical potential $\mu$ and the inverse temperature $\beta=1 / T$ (reduced values are assumed henceforward). One thus has

$$
\begin{aligned}
h & =\mu-\mu_{\mathrm{c}}+r\left(\beta-\beta_{\mathrm{c}}\right), \\
\tau & =\beta_{\mathrm{c}}-\beta+s\left(\mu-\mu_{\mathrm{c}}\right),
\end{aligned}
$$

where $r$ and $s$ are the field mixing parameters which define the mapping. Of course relations (3.1) are valid only in the vicinity of the CP. The conjugate scaling operators $\mathcal{M}$ and $\mathcal{E}$ are then defined as

$$
\begin{aligned}
\langle\mathcal{M}\rangle & =\frac{1}{V} \frac{\partial}{\partial h} \ln \Xi=\frac{1}{1-s r}(\langle\rho\rangle-s\langle u\rangle), \\
\langle\mathcal{E}\rangle & =\frac{1}{V} \frac{\partial}{\partial \tau} \ln \Xi=\frac{1}{1-s r}(\langle u\rangle-r\langle\rho\rangle),
\end{aligned}
$$

where $\Xi$ is the GC partition function of the RPM, $\rho$ is the total number density, and $u$ is the internal energy per unit volume. Brackets $\langle\ldots\rangle$ denote GC averages. $\mathcal{M}$ is the order parameter (magnetization) of the magnetic system associated with the fluid and $\mathcal{E}$ is its magnetic energy. $\mathcal{E}$ should be invariant under the transformations $(\mathcal{M}, h) \rightarrow(-\mathcal{M},-h)$ for appropriate values of $s$ and $r$. In this framework the coexistence curve is therefore defined by the equation $h=0$.

The revised scaling of Rehr and Mermin implies the analyticity of the coexistence chemical potential $\mu(T)$ at $T_{\mathrm{c}}^{*}$. Although this is the case for some peculiar lattice gas models with "hidden" symmetries there is no reason that in general, for fluid systems $\mu(T)$ should lack a singularity as recognized already by Rehr and Mermin [23] and emphasized more recently by Fisher and co-workers [40-42] .

\subsection{The scaling hypothesis}

A central role in the subsequent fss analysis is played by the $\mathrm{GC}$ joint distribution $\mathcal{P}_{\mathrm{L}}(\mathcal{M}, \mathcal{E}) \propto \mathcal{P}_{\mathrm{L}}(\rho, u)$ for the scaling operators $\mathcal{M}$ and $\mathcal{E}$. Following BW $[20-22]$ we will assume that, in the immediate vicinity of the $\mathrm{CP}, \mathcal{P}_{\mathrm{L}}(\mathcal{M}, \mathcal{E})$ obeys to the following scaling law:

$$
\begin{aligned}
\mathcal{P}_{\mathrm{L}}(\mathcal{M}, \mathcal{E})= & a_{\mathcal{M}}^{-1} a_{\mathcal{E}}^{-1} L^{d-y_{\tau}} L^{d-y_{h}} \widetilde{\mathcal{P}}\left(a_{\mathcal{M}}^{-1} L^{d-y_{h}} \delta \mathcal{M}\right. \\
& \left.\ldots a_{\mathcal{E}}^{-1} L^{d-y_{\tau}} \delta \mathcal{E}, a_{\mathcal{M}} L^{y_{h}} h, a_{\mathcal{E}} L^{y_{\tau}} \tau, a_{i} L^{y_{i}}, \ldots\right)
\end{aligned}
$$


where $L$ are the linear dimensions of the system (taken as $V^{1 / 3}$, where $V$ is the volume of the simulation box, either a cube or a hypersphere). I have denoted by $\delta \mathcal{M} \equiv \mathcal{M}-\langle\mathcal{M}\rangle_{\mathrm{c}}$ and $\delta \mathcal{E} \equiv \mathcal{E}-\langle\mathcal{E}\rangle_{\mathrm{c}}$ the deviations of the scaling operators from their value at criticality. The cornerstone of this scaling hypothesis is that the function $\widetilde{\mathcal{P}}$ which enters equation (3.3) is universal in the sense that it depends only upon the universality class of the model and on the type of geometry considered. The constants $a_{\mathcal{M}}, a_{\mathcal{E}}$, and $a_{i}$ are system dependent constants which are defined in such a way that $\widetilde{\mathcal{P}}$ has unit variance. Finally, the renormalization exponents $y_{h}, y_{\tau}$, and $y_{i}$ which enter equation (3.3) are defined as

$$
y_{h}=d-\beta / \nu, \quad y_{\tau}=1 / \nu, \quad y_{i}=-\theta / \nu
$$

in terms of the usual critical exponents:

- $\beta$ exponent of the ordering field, i.e. $<\delta \mathcal{M}>\sim|\tau|^{\beta}$ for $T^{*}<T_{\mathrm{c}}^{*}$ at $h=0$;

- $\nu$ exponent of the correlation length, i.e. $\xi \sim|\tau|^{-\nu}$;

- $\theta$ Wegner's correction-to-scaling exponent (first irrelevant exponent).

The scaling hypothesis (3.3) was established on a solid RG basis for Ising-like systems [24] and received substantial support from MC studies [25]. We stress once again that the coexistence curve is determined in this approach by the condition $h=0$ and that, at coexistence, the order parameter distribution $\mathcal{P}_{\mathrm{L}}(\mathcal{M})$ should be an even function of $\mathcal{M}$. In practice this symmetry requirement can be satisfied by tuning the two parameters $(\mu, s)$ at a given $\beta$. We now concentrate our attention on the scaling behavior of the histogram $\mathcal{P}_{\mathrm{L}}(\mathcal{M})$.

\subsection{The matching procedure}

Integrating both sides of equation (3.3) over $\mathcal{E}$ one finds that, along the coexistence line $h=0$ one has

$$
\mathcal{P}_{\mathrm{L}}(\mathcal{M})=a_{\mathcal{M}}^{-1} L^{d-y_{h}} \widetilde{\mathcal{P}}\left(a_{\mathcal{M}}^{-1} L^{d-y_{h}} \delta \mathcal{M}, a_{\mathcal{E}} L^{y_{\tau}} \tau, a_{i} L^{y_{i}}\right)
$$

where, in the r.h.s. the dependence of the universal function $\widetilde{\mathcal{P}}$ upon $h$ has been discarded for clarity. Let us define now $x=a_{\mathcal{M}}^{-1} L^{d-y_{h}} \delta \mathcal{M}$, then, assuming $\tau \sim 0$ and $L \sim \infty$ a Taylor expansion of equation (3.5) yields

$$
\begin{aligned}
\mathcal{P}_{\mathrm{L}}(\mathcal{M})= & a_{\mathcal{M}}^{-1} L^{d-y_{h}}\left[\widetilde{\mathcal{P}}^{*}(x)+a_{\mathcal{E}} L^{y_{\tau}} \tau \widetilde{\mathcal{P}}_{1}^{*}(x)\right. \\
& \left.+a_{\mathcal{E}}^{\prime 2} L^{2 y_{\tau}} \tau^{2} \widetilde{\mathcal{P}}_{2}^{*}(x)+\ldots+a_{i} L^{y_{i}} \widetilde{\mathcal{P}}_{3}^{*}(x)+\ldots\right]
\end{aligned}
$$

where the various $\widetilde{\mathcal{P}}^{*}$ entering the r.h.s. are universal functions. Note that, for $L=$ $\infty$ the normalized ordering field distribution $\mathcal{P}_{\mathrm{L}}(\mathcal{M})$ collapses onto an universal function $\widetilde{\mathcal{P}}^{*}(x)$ at $\tau=0$. For $L$ finite but large $\mathcal{P}_{\mathrm{L}}(\mathcal{M})$ collapses approximately onto $\widetilde{\mathcal{P}}^{*}(x)$ at some apparent $\tau_{\mathrm{L}} \propto L^{-y_{\tau}+y_{i}}$. Since for $h=0$ one has $\tau \propto \beta-\beta_{c}$ then the 
matching of the histogram $\mathcal{P}_{\mathrm{L}}(\mathcal{M})$ onto the universal function $\widetilde{\mathcal{P}}^{*}(x)$ should occur at some apparent temperature $T_{\mathrm{c}}^{*}(L)$ scaling with system size as

$$
T_{\mathrm{c}}^{*}(\infty)-T_{\mathrm{c}}^{*}(L) \propto L^{-(\theta+1) / \nu}+\ldots,
$$

where $T_{\mathrm{c}}^{*}(\infty)$ denotes the infinite volume limit of the critical temperature.

\subsection{Technical details}

To assess the critical behavior and the critical parameters of the system, we need, in a first step, to locate the coexistence curve $h=0$. At a given temperature $\beta$ close to $\beta_{\mathrm{c}}$ the ordering distribution function $\mathcal{P}_{\mathrm{L}}(\mathcal{M})$ depends solely on the chemical potential $\mu$ and the mixing parameter $s$. At coexistence, the value of $(\mu, s)$ can be obtained unambiguously by requiring that $\mathcal{P}_{\mathrm{L}}(\mathcal{M})$ is symmetric in $\mathcal{M}-\langle\mathcal{M}\rangle[22]$. Tuning at will $\mu$ and $s$ at given $\beta$ requires to know the joint histogram $\mathcal{P}_{\mathrm{L}}(\mathcal{M}, \mathcal{E}) \propto \mathcal{P}_{\mathrm{L}}(\rho, u)$ for a continuous set of values of $\mu$ at a given $\beta$. Moreover, since this analysis must be performed at different $\beta$ one needs in fact to know $\mathcal{P}_{\mathrm{L}}(\mathcal{M}, \mathcal{E})$ for a continuous set of values of $(\beta, \mu)$ in the critical region. This technical difficulty is circumvented by using the multiple histogram reweighting proposed by Ferrenberg and Swensen [26-28]. With this method one can obtains $\mathcal{P}_{\mathrm{L}}^{\beta, \mu}(\rho, u)$ for a continuous set of values of $(\beta, \mu)$ from the knowledge of $R$ histograms $\mathcal{P}_{\mathrm{L}}^{\beta_{i}, \mu_{i}}(\rho, u), i=1, \ldots, R$ obtained by performing $R$ distinct MC simulations in the $R$ (neighbor) thermodynamic states $\left(\beta_{i}, \mu_{i}\right)$.

Since the precision of the simulations of fluid systems has still not reached that obtained in the MC simulations of Ising like systems it is impossible to construct ex nihilo the fixed point universal distribution $\widetilde{\mathcal{P}}^{*}(x)$. In $[29,30]$ our attempts to match $\mathcal{P}_{\mathrm{L}}(\mathcal{M})$ on $\widetilde{\mathcal{P}}^{*}(x)$ were realized by using the estimate of $\widetilde{\mathcal{P}}_{\text {is }}^{*}(x)$ made by Hilfer and Wilding [32] for the 3D Ising model. Two new -and better- estimates of $\widetilde{\mathcal{P}}_{\text {is }}^{*}(x)$ obtained by Tsypin and Blöte [33] for the 3D Ising model and the spin-1 BlumeCapel model were considered in [31]. The discussion is postponed to next section.

\section{Results}

\subsection{General discussion}

It turns out that the field mixing parameter $s$ of the RPM is practically independent of the temperature and of the size $L$ of the system. Its magnitude, $s \sim-1.46$ [29-31], is much higher than for neutral fluids (typically $s \sim 0.02$ for square well or Lennard-Jones fluids [34]) which explains the large dissymmetry of the liquid-vapor coexistence curve of the RPM.

The collapse of the ordering operator distribution $\mathcal{P}_{\mathrm{L}}(\mathcal{M})$ onto the universal ordering distribution $\widetilde{\mathcal{P}}_{\text {is }}^{*}(x)$ given by the Blume-Capel model [33] is depicted in figure 1 for four different values of the volume ranging from $V / \sigma^{3}=5000$ to $V / \sigma^{3}=$ 40000 , i.e. up to a linear size $L / \sigma=34$. At volume $V / \sigma^{3}=5000$ a mismatch 


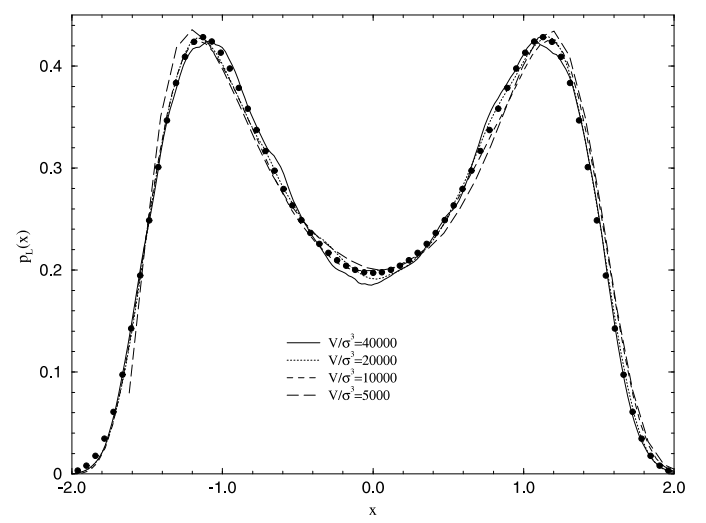

Figure 1. Collapse of the ordering distribution $\mathcal{P}_{\mathrm{L}}(\mathcal{M})$ onto the universal Ising ordering distribution $\widetilde{\mathcal{P}}_{\text {is }}^{*}(x)$ for $V / \sigma^{3}=5000, T_{\mathrm{c}}^{*}(L)=0.004934, s=-1.45$; $V / \sigma^{3}=10000, T_{\mathrm{c}}^{*}(L)=0.004926, s=-1.465 ; V / \sigma^{3}=20000, T_{\mathrm{c}}^{*}(L)=0.004921$, $s=-1.47 ;$ and $V / \sigma^{3}=40000, T_{\mathrm{c}}^{*}(L)=0.004922, s=-1.43 . \widetilde{\mathcal{P}}_{\mathrm{is}}^{*}(x)$ (solid circles) if the MC result of Tsypin and Blöte ([33]) obtained for the Blume-Capel model. The scaling variable is $x=a_{\mathcal{M}}^{-1} L^{\beta / \nu} \delta \mathcal{M}$ where $a_{\mathcal{M}}$ is chosen in such a way that $\mathcal{P}_{\mathrm{L}}(x)$ has unit variance.
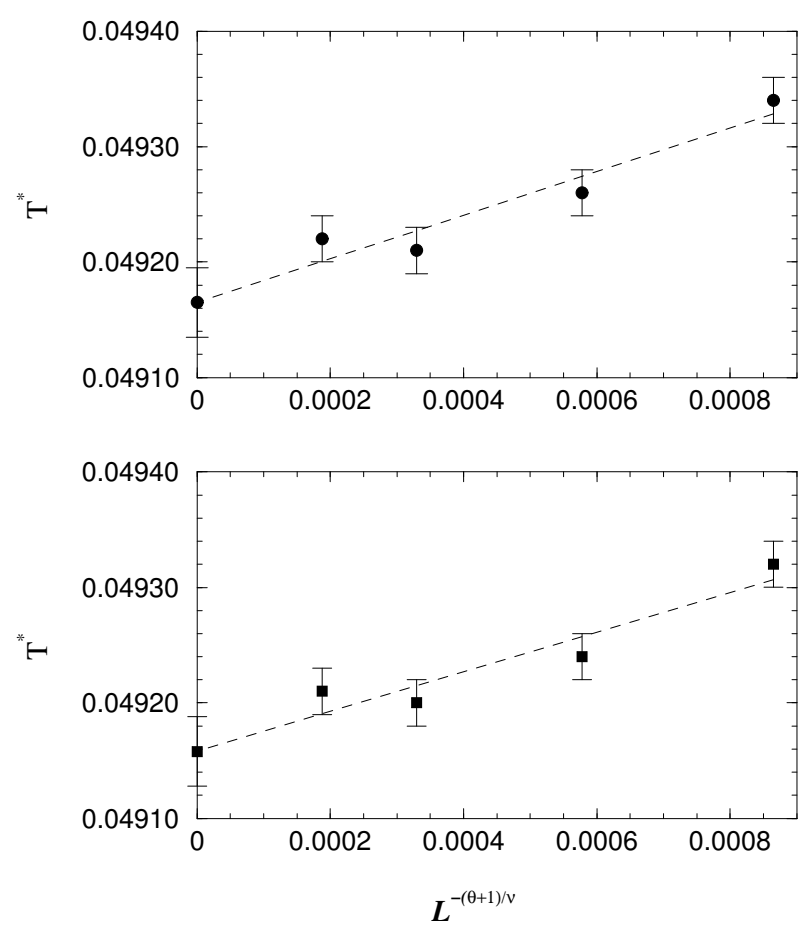

Figure 2. The apparent critical temperature $T_{\mathrm{c}}^{*}(L)$ as a function of $L^{-(\theta+1) / \nu}$ with $\theta=0.53, \nu=0.630$ obtained by matching the universal ordering distribution calculated for the Blume-Capel model (top) and the Ising model (bottom). Extrapolating by linear least square fit to the infinite volume limit yields $T_{\mathrm{c}}^{*}(\infty)=0.04917 \pm 0.00002$ (top) and $T_{\mathrm{c}}^{*}(\infty)=0.04916 \pm 0.00002$ (bottom). 
is observed at the lowest values of $\mathcal{M}$ due to an inadequate sampling of the low density configurations at small volume. The overall good agreement leads us to conclude that the universality class of the RPM is that of the 3D Ising model.

The reduced apparent critical temperature $T_{\mathrm{c}}^{*}(L)$ versus the size $L$ of the system (in reduced units) has been plotted in figure 2. Depending on the choice made for the universal ordering distribution $\widetilde{\mathcal{P}}_{\text {is }}^{*}(x)$ one obtains two sets of values of $T_{\mathrm{c}}^{*}(L)$ from which $T_{\mathrm{c}}^{*}(\infty)$ can be obtained by making use of equation (3.7). One obtains $T_{\mathrm{c}}^{*}(\infty)=0.04917 \pm 0.00002$ using $\widetilde{\mathcal{P}}_{\text {is }}^{*}(x)$ derived from the Blume-Capel model and $T_{\mathrm{c}}^{*}(\infty)=0.04916 \pm 0.00002$ using $\widetilde{\mathcal{P}}_{\text {is }}^{*}(x)$ obtained for the 3D-Ising model. The approximate $\widetilde{\mathcal{P}}_{\text {is }}^{*}(x)$ of Hilfer and Wilding yields slightly different results. Note that in all cases we have used the Ising values $\nu=0.630$ [35] and $\theta=0.53$ [36] of the critical exponents.

The previous analysis merely establishes the compatibility of the MC data with an Ising-like criticality. One can try to go beyond by considering the scaling behavior of the Binder cumulant

$$
Q_{\mathrm{B}}(L)=\frac{\left\langle\delta \mathcal{M}^{2}\right\rangle_{\mathrm{L}}^{2}}{\left\langle\delta \mathcal{M}^{4}\right\rangle_{\mathrm{L}}}
$$

As a consequence of the scaling hypothesis (3.6) one can show that, at coexistence $(h=0), Q_{\mathrm{B}}(L)$ should scale with system size as

$$
\begin{aligned}
Q_{\mathrm{B}}(L)= & Q_{\mathrm{c}}+q_{1}\left(\beta-\beta_{\mathrm{c}}\right) L^{1 / \nu}+q_{2}\left(\beta-\beta_{\mathrm{c}}\right)^{2} L^{2 / \nu} \\
& +q_{3}\left(\beta-\beta_{\mathrm{c}}\right)^{3} L^{3 / \nu} \ldots+b_{1} L^{y_{i}}+\ldots,
\end{aligned}
$$

where the last term takes into account contributions from irrelevant fields and $q_{1}, q_{2}$, $q_{3}$, and $b_{1}$ are non-universal constants. If the contribution of irrelevant fields could be neglected then the curves $Q_{\mathrm{B}}(L)$ would intersect at the fixed point $Q_{\mathrm{c}}$. As apparent in figure 3 this is clearly not the case and corrections to scaling must be taken into account.

Recall that for the 3D-Ising model the fixed point value is $Q_{\mathrm{c}}=0.623$ [37] and that the exponent of the correlation length has the value $\nu=0.630$ [35]. We have attempted to fit all our MC data with equation (4.2). If all the parameters in the RHS of equation (4.2) are kept free such an ambitious fit turns out to be impossible. Various other less satisfactory strategies can be considered however.

- Fixing $\beta_{\mathrm{c}}=1 / 0.04917, y_{i}=-\theta / \nu=-0.84$ and leaving free all the other parameters one finds a fit better than 1 per cent and $Q_{\mathrm{c}}=0.63 \pm 0.01, \nu \sim$ $0.66 \pm 0.03$.

- Conversely, fixing $Q_{\mathrm{c}}=0.623$ and $\theta=0.53$ one obtains $\beta_{\mathrm{c}}=1 / 0.04918$ and $\nu \sim 0.63 \pm 0.03$.

The variations of $Q_{\mathrm{B}}(L)$ as a function of $\beta$ for the different volumes is shown in figure 3. Although there is considerable spread in the intersection points due to correction-to-scaling contributions, the corresponding values of $Q_{\mathrm{c}}$ are close to the 


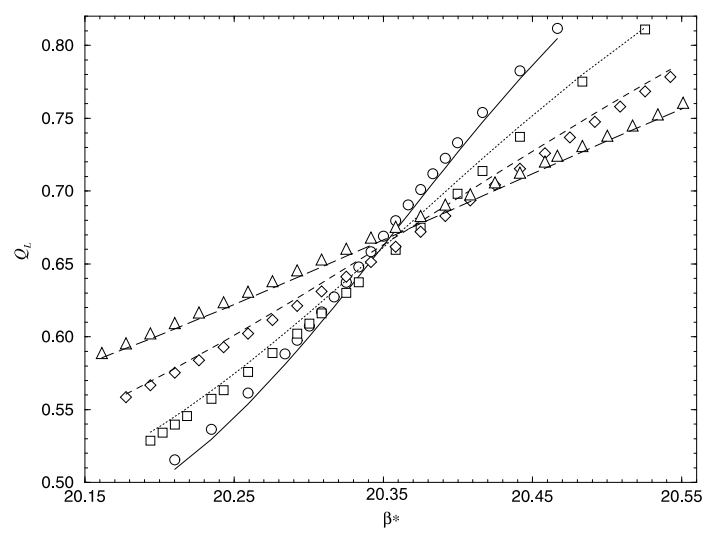

Figure 3. Variation of $Q_{\mathrm{B}}(L)$ as a function of the inverse temperature $\beta$ for the different volumes considered in [31]. From top to bottom $V / \sigma^{3}=$ 40000, 20000, 10000, and 5000 respectively. The symbols are the MC data and the lines are the fits obtained by means of equation (4.2).

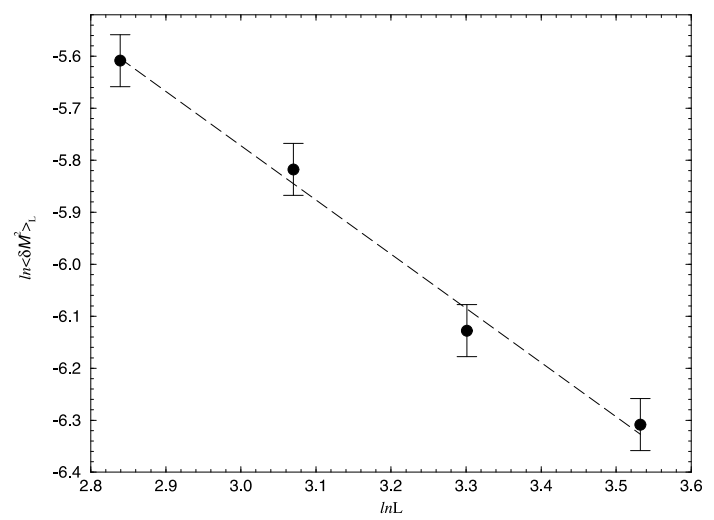

Figure 4. Variations of $\ln \left\langle\delta \mathcal{M}^{2}\right\rangle$ at $T_{\mathrm{c}}^{*}(L)$ as a function of $\ln L$. The slope of the linear least square fit is $2 \beta / \nu=1.04$.

Ising value $Q_{\mathrm{c}}=0.623$ and permit to rule out mean field behavior (i.e. $Q_{\mathrm{c}}=0.457$ [38]).

Further support for Ising criticality is provided by the behavior of $\left\langle\delta \mathcal{M}^{2}\right\rangle$ at $T_{\mathrm{c}}^{*}(L)$. According to the scaling hypothesis (3.6) it should scale as $L^{2 \beta / \nu}$ with system size. From the slope of the curve displayed in figure 4 one obtains $\beta / \nu=0.52$ in accord with the 3D Ising value (0.517) and in clear contrast with the classical value 1.

In summary, our fss analysis leads to an estimate of the critical exponents $\nu$ and $\beta / \nu$ and the Binder cumulant $Q_{\mathrm{c}}$ based on the sole knowledge of the critical temperature and the renormalization exponent $\theta$. Within the numerical uncertainties these values are compatible with Ising-like criticality. Our conclusion is that the RPM, as ordinary neutral fluids, belongs to the universality class of the Ising model.

A complete discussion of our MC data is out of the scope of the present paper and can be found in [31]. For completeness I give below the values obtained for 
the critical temperature, chemical potentials and densities (the critical pressure is largely unknown):

- $T_{\mathrm{c}}^{\star}=0.04917 \pm 0.00002$,

- $\rho_{\mathrm{c}}^{\star}=0.080 \pm 0.005$,

- $\mu_{\mathrm{c}}^{\star}=-13.600 \pm 0.005$.

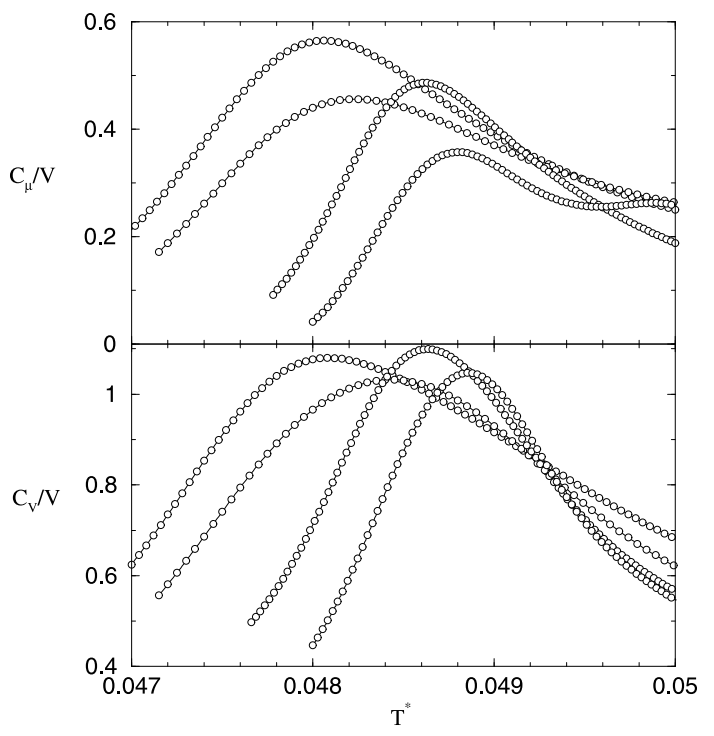

Figure 5. Variations of the total specific heat at constant volume $C_{\mathrm{V}} / V$ and the contribution $C_{\mu} / V$ with temperature along the locus $\chi_{N N N}=0$ at volumes $V / \sigma^{3}=5000,10000,20000$, and 40000 (from left to right).

\subsection{The specific heat}

The revised scaling theory of Rehr and Mermin which is the framework of our fss analysis is however not the most general scaling theory which can be proposed for a fluid system lacking the "particle-hole" symmetry. Its main weakness, as recognized already by Rehr and Mermin [23], Yang and Yang [39], and more recently by Fisher and co-workers [40-42], is that it assumes the analyticity of the chemical potential at coexistence $\mu(T)$ at the critical point. The more general scaling assumption should yield singularities for both $\mu(T)$ and $p(T)$ as $T^{*} \rightarrow T_{\mathrm{c}}^{*}$. Let us examine the effects of these singularities on the behavior of the specific heat capacity at constant volume $C_{\mathrm{V}}$. In the two phase region it can be rewritten as [39]

$$
C_{\mathrm{V}}=\left.V T \frac{\partial^{2} p}{\partial T^{2}}\right|_{\mathrm{V}}-\left.N T \frac{\partial^{2} \mu}{\partial T^{2}}\right|_{\mathrm{V}}=C_{\mathrm{p}}+C_{\mu}
$$

where $C_{\mathrm{p}}$ (not to be confused with the specific heat capacity at constant pressure) and $C_{\mu}$ (not to be confused with the specific heat capacity at constant chemical 
potential) denote the two contributions to $C_{\mathrm{V}}$. I stress that, in equation (4.3) $p(T)$ and $\mu(T)$ denote the pressure and the chemical potential at coexistence. The formula can be used for any density $\rho_{\mathrm{g}}(T)<\rho<\rho_{\mathrm{l}}(T)$ within the two phase region $\left(\rho_{\mathrm{g}}(T)\right.$ and $\rho_{1}(T)$ being the densities of the gas and the liquid at coexistence respectively). In the revised scaling theory only $C_{\mathrm{p}}$ diverges as $\left|T^{*}-T_{\mathrm{c}}^{*}\right|^{-\alpha}$ whereas one expects a divergence of both $C_{\mathrm{p}}$ and $C_{\mu}$ (both as $\left|T^{*}-T_{\mathrm{c}}^{*}\right|^{-\alpha}$ ). In figure $5 \mathrm{I}$ display the curves $C_{\mu}(T)$ and $C_{\mathrm{V}}(T)$ along the locus

$$
\chi_{N N N} \propto\left\langle(N-\langle N\rangle)^{3}\right\rangle=0
$$

for the four volumes considered in our last MC simulations [31]. Although the peak positions shift correctly as $\propto L^{-1 / \nu}$ with system size, in accord with fss theory $[18,19]$, there is no detectable scaling of the heights of the peaks which should scale as $L^{\alpha / \nu}$ with $L$. These observations corroborate similar results obtained by Valleau and Torrie $[43,44]$. In particular $C_{\mu}$ does not show any anomaly which should challenge the use of equations (3.1) for the scaling fields. A possible explanation for the non singular behavior of $C_{\mathrm{V}}(T)$ is that the amplitude of the singular term in $C_{\mathrm{V}}(T)$ is small in the RPM and that the specific heat is dominated by its regular part. Note however that the peak heights in $C_{\mathrm{V}}(T) / V$ would scale, assuming Ising value for $\alpha$ only by a factor $2^{\alpha / \nu} \sim 1.12$ when doubling the linear dimensions of the system. It is possible that such a small effect is not detectable within the statistical uncertainties of our calculations.

\section{Conclusion}

In this paper which resumes my talk at the Lviv NATO workshop I have described recent attempts to elucidate the nature of the critical behavior of the RPM model for ionic fluids, prototype of a system governed by long range Coulomb interactions by means of MC simulations. After endeavor over more than a decade we have now reached a point where we can claim confidently that the RPM belongs to the same universality class as the 3D Ising model. The critical values of non-universal quantities such as the temperature and the chemical potential were established with a high accuracy whereas the uncertainties on the critical density are more significant, and the critical pressure is unknown.

The behavior of the constant volume specific heat gives no indication of the expected $L^{\alpha / \nu}$ scaling of the peak height within the range of system sizes considered in the most recent simulations. Recent investigations of Camp and co-workers [45] where differences in the behavior of $C_{\mathrm{V}}$ in the canonical and the $\mathrm{GC}$ ensemble are reported have emphasized this problem. At the moment it is difficult to explain this unexpected behavior of the specific heat.

I have only discussed the properties of the continuous version of the RPM. The phase diagram of the various lattice versions of the model is in fact more complex $[46,47]$ and was not described here due to a lack of place. I have also excluded from my presentation assymetric versions, either in charge or/and in size, of the 
continuum or lattice versions of the primitive model. The interested reader should consult recent works of Panagiotopoulos et al. [48-50] and de Pablo et al. [51-53].

\section{Acknowledgments}

I thank the organizers of the NATO workshop "Ionic Soft Matter" held in Lviv, Ukraine in April 2004, Pr. D.Henderson and Pr. M.Holovko for having invited me to give a talk. It is a pleasure to acknowledge many scientific discussions with Pr. I.M.Mryglod, O.Patsahan, J.-P.Badiali, P.J.Camp, W.Schröer, and J.Stafiej.

\section{References}

1. Weingärtner H., Schröer W. // Adv. Chem. Phys., 2001, vol. 116, p. 1.

2. Fisher M.E. // J. Stat. Phys., 1994, vol. 75, p. 1.

3. Stell G. // J. Stat. Phys., 1995, vol. 78, p. 197.

4. Fisher M.E. // J. Phys.: Condens. Matter, 1996, vol. 8, p. 9103.

5. Stell G. // J. Phys.: Condens. Matter, 1996, vol. 8, p. 9329.

6. Vorontsov-Vel' Yaminov P.N., Chasovkikh B.P. // High. Temp., 1975, vol. 13, p. 1071.

7. Chasovkikh B.P., Vorontsov-Vel' Yaminov P.N. // High. Temp., 1976, vol. 14, p. 1174.

8. Valleau J. // J. Chem. Phys., 1991, vol. 95, p. 584.

9. Panagiotopoulos A.Z. // Fluid Phase Equilibria, 1992, vol. 76, p. 97.

10. Panagiotopoulos A.Z. // Mol. Phys., 1987, vol. 61, p. 813.

11. Orkoulas G., Panagiotopoulos A.Z. // J. Chem. Phys., 1994, vol. 101, p. 1452.

12. Caillol J.-M. // J. Chem. Phys., 1994, vol. 100, p. 2169.

13. Caillol J.-M. // J. Phys.: Condens. Matter, 1994, vol. 6, p. A171.

14. Caillol J.-M., Weis J.-J. // J. Chem. Phys., 1995, vol. 102, p. 7610.

15. Brush S.G., Sahlin H.L., Teller E. // J. Chem. Phys., 1966, vol. 45, p. 2102.

16. de Leeuw S.W., Perram S.W., Smith E.R. // Proc. R. Soc. London A, 1980, vol. 373, p. 27.

17. Caillol J.-M. // J. Chem. Phys., 1999, vol. 111, p. 6528.

18. Privman V., ed. Finite Size Scaling and Numerical Simulation in Statistical Systems. Singapore, World Scientific, 1990.

19. Cardy J.L., ed., Finite Size Scaling. Amsterdam, North Holland, 1988.

20. Bruce A.D., Wilding N.B. // Phys. Rev. Lett., 1992, vol. 68, p. 193.

21. Wilding N.B., Bruce A.D. // J. Phys.: Condens. Matter, 1992, vol. 4, p. 3087.

22. Wilding N.B. // Phys. Rev. E, 1995, vol. 52, p. 602.

23. Rehr J.J., Mermin N.D. // Phys. Rev. A, 1973, vol. 8, p. 472.

24. Bruce A.D. // J. Phys. C: Solid State Phys. 1981, vol. 14, p. 3667.

25. Nicolaides D., Bruce A.D. // J. Phys. A: Math. Gen. 1988, vol. 21, p. 233.

26. Ferrenberg A.M., Swendsen R.R. // Phys. Rev. Lett., 1988, vol. 61, p. 2635.

27. Ferrenberg A.M., Swendsen R.R. // Phys. Rev. Lett., 1989, vol. 63, p. 1195.

28. Deutsch H.-P. // J. Stat. Phys., 1992, vol. 67, p. 1039.

29. Caillol J.-M., Levesque D., Weis J.-J. // Phys. Rev. Lett., 1996, vol. 77, p. 4039.

30. Caillol J.-M., Levesque D., Weis J.-J. // J. Chem. Phys., 1997, vol. 107, p. 1565.

31. Caillol J.-M., Levesque D., Weis J.-J. // J. Chem. Phys., 2002, vol. 116, p. 10794.

32. Hilfer R., Wilding N.B. // J. Phys. A, 1995, vol. 28, p. L281. 
33. Tsypin M.M., Blöte H.W.J. // Phys. Rev. E, 2000, vol. 62, p. 73.

34. Caillol J.-M. // J. Chem. Phys., 1998, vol. 109, p. 4885.

35. Ferrenberg A.M., Landau .D.P. // Phys. Rev. B, 1991, vol. 44, p. 5081.

36. Chen J.H., Fisher M.E., Nickel B.G. // Phys. Rev. Lett., 1982, vol. 48, p. 630.

37. Blöte H.W.J., Luijten E., Heringa J.R. // J. Phys. A, 1995, vol. 28, p. 6289.

38. Luijten E., Blöte H.W.J. // Phys. Rev. Lett., 1996, vol. 76, p. 1557.

39. Yang C.N., Yang C.P. // Phys. Rev. Lett., 1964, vol. 13, p. 303.

40. Fisher M.E., Orkoulas G. // Phys. Rev. Lett., 2000, vol. 85, p. 696.

41. Orkoulas G., Fisher M.E., Ustün C. // J. Chem. Phys., 2000, vol. 113, p. 7530.

42. Orkoulas G., Fisher M.E., Panagiotopoulos A.Z. // Phys. Rev. E, 2001, vol. 63, p. 051507.

43. Valleau J., Torrie G. // J. Chem. Phys., 1998, vol. 108, p. 5169.

44. Valleau J., Torrie G. // J. Chem. Phys., 2002, vol. 117, p. 3305.

45. Daub C.D., Camp P.J., Patey G.N. // J. Chem. Phys., 2003, vol. 118, p. 4164.

46. Luijten E., Fisher M.E., Panagiotopoulos A.Z. // J. Chem. Phys., 2001, vol. 114, p. 5468.

47. Panagiotopoulos A.Z. // J. Chem. Phys., 2002, vol. 116, p. 3007.

48. Panagiotopoulos A.Z., Fisher M.E. // Phys. Rev. Lett., 2002, vol. 88, p. 045701-1.

49. Cheong D.W., Panagiotopoulos A.Z. // J. Chem. Phys., 2003, vol. 119, p. 8526.

50. Romero-Enrique J.M., Rull L.F., Panagiotopoulos A.Z. // Phys. Rev. E, 2003, vol. 66, p. 041204.

51. Yan Q., de Pablo J.J. // J. Chem. Phys., 2001, vol. 114, p. 177.

52. Yan Q., de Pablo J.J. // Phys. Rev. Lett., 2002, vol. 88, p. 095504-1.

53. Yan Q., de Pablo J.J. // J. Chem. Phys., 2002, vol. 116, p. 2697. 


\section{Моделювання методом Монте Карло критичних властивостей примітивної моделі електролітів}

\section{Ж.-М.Кайоль}

Лабораторія теоретичної фізики, Університет Парижу XI, Орсей, Франція

Отримано 16 серпня 2004 р., в остаточному вигляді 25 серпня $2004 \mathrm{p}$.

Представлені результати моделювання методом Монте Карло критичної точки примітивної моделі електролітів. Розглянуто тільки неперервну версію моделі. Аналіз скінченомірного скейлінгу, що базується на процедурі Брюса-Вілдінга дає критичні індекси, які узгоджуються 3 класом універсальної тривимірної моделі Ізінга. Відмічено аномалію в скейлінговій поведінці питомої теплоємності.

Ключові слова: примітивна модель, іонна критичність, моделювання методом Монте Карло

PACS: 05.70.Jk, 05.10.-a, 64.70.Fx, 61.20.-p 